\title{
Prevalence and Resistance Pattern of Pseudomonas aeruginosa Isolated from Surface Water
}

\author{
Marufa Nasreen'1, Animesh Sarker'1, M. A. Malek², Md. Ansaruzzaman³, \\ Mahububur Rahman ${ }^{3}$ \\ ${ }^{1}$ Department of Biotechnology and Genetic Engineering, Mawlana Bhashani Science and Technology \\ University, Santosh, Tangail, Bangladesh \\ ${ }^{2}$ Department of Microbiology, University of Dhaka, Dhaka, Bangladesh \\ ${ }^{3}$ International Centre for Diarrheal Diseases Research, Dhaka, Bangladesh \\ Email: nasreen.marufa@gmail.com
}

Received 2 January 2015; accepted 21 January 2015; published 30 January 2015

Copyright (C) 2015 by authors and Scientific Research Publishing Inc.

This work is licensed under the Creative Commons Attribution International License (CC BY).

http://creativecommons.org/licenses/by/4.0/

c) (i) Open Access

\section{Abstract}

Pseudomonas aeruginosa is one of the most common pathogenic bacteria, frequently found in different environmental samples. The prevalence of multidrug resistant isolates has become an alarming concern for both patients and their surroundings. The present study was carried out to record prevalence of $P$. aeruginosa in surface water of Dhaka city and to screen their antibiotic resistance pattern. The study was also extended to typing of resistant isolates according to extended spectrum beta lactamase production. Hereby, Kirby-Bauer method was applied to test antibiotic sensitivity according to Clinical and Laboratory Standards Institute. Then, the Ampicillin resistant isolates were screened for ESBL production by Double Disk Synergy Test (DDST). In these prospects, 52 water samples were tested, of which 32 were found positive for $P$. aeruginosa isolates. Hundred percent of the positive isolates were found to Ampicillin (AMP) resistant followed by $\mathbf{9 3 . 7 \%}$ to both Tetracycline and Gentamycin and $71.8 \%$ to Co-triimoxazole. P. aeruginosa is completely susceptible to third generation antibiotics ciprofloxacin, Imipenem and Aztreonam followed by moderately susceptible to Polymyxin-B (78.2\%) and Colistin (87.5\%). According to DDST, all of the susceptible isolates were found positive for AMC type beta-lactamase production. It is evident from this study that the surface water is contaminated with antibiotic resistant $P$. aeruginosa and that through the water systems antibiotic resistance can be transferred to humans and animals. So, appropriate and rationale use of antibiotic should be applied to minimize the emergence of multidrug isolates to environment. 


\section{Keywords}

\section{P. aeruginosa, Beta-Lactamase, DDST, Multidrug Resistant}

\section{Introduction}

Pseudomonas aeruginosa is a clinically important gram negative bacterium, which is responsible for a variety of systemic infections like urinary tract infections, respiratory system infections, gastrointestinal infections, dermatitis, bacteremia, soft tissue infections, bone and joint infections [1]. Due to its nominal nutritional requirement, $P$. areuginosa has the ability to survive in soil, plant surfaces, waste water, moist environment, surface water, or even on inert materials [2]. Using life support equipments, cosmetics, dilute antiseptics even through the washing liquids and soaps, it can be transmitted person to person [3] [4]. However, P. aeruginosa is mostly acquired from environment and spread person-to-person rarely [5].

$P$. arenginosa is highly ubiquitous in water system and capable to acquire antibiotic resistance due to its low outer membrane permeability and extensive efflux pump system [6]-[8]. The overall prevalence of antibiotic resistant $P$. aeruginosa is increasing, with up to $10 \%$ of global isolates found to be multi-drug resistance [9]. It is recognized as the second leading cause of gram negative nosocomial infection and a major treatment challenge for Pseudomonas aeruginosa [10].

Dhaka is one of the densely populated cities in the world. There are a huge number of hospitals and clinics in Dhaka City where different antibiotics are used frequently. After their use in various treatments such as medicine, veterinary medicine and growth promoters in animal husbandry, fish farming and other fields' antibiotics are released into the water environment. Frequent use of antibiotics in treatments plays an important role in the emergence of resistant bacteria [11]. Moreover, most of the antibiotic producing pharmaceuticals are located in these areas. Only few of them maintain the guidelines to dispose effluents in ponds, rivers and other water systems.

Bacteria resistant to antibiotics have been detected in different compartments such as waste water, surface water, ground water, sediments and soils. It is necessary to study the presence of clinically important bacteria in environment and their antibiotic resistance pattern. In the past two decades, the emergence of resistance to antimicrobial agents has been repented as one of the most important clinical crisis [12]. Due to the production of a variety of beta-lactamases, alterations in the penicillin-binding proteins and outer membrane permeability, and combinations of multiple mechanisms of resistance, the gram negative bacteria have acquired resistance to antibiotics such as extended-spectrum Cephalosporins, Monobactams, Carbapenems and beta-lactam-beta-lactamase inhibitor combinations [13]. Extended-spectrum beta-lactamases (ESBLs) are an important reason of bacterial resistance throughout the world [14]. Microbiologists, clinicians, infection control Professionals and scientists engaged in finding new antimicrobial agents are now facing distinctive challenge to ESBL producing isolates. ESBLs are also able to hydrolyze 3rd and 4th generation Cephalosporins and Monobactams. ESBL producing strains are only inhibited by beta-lactamase inhibitors, clavulanic acid, sulbactam and tazobactam [15] [16].

The objectives of the present investigations were to assess the prevalence of $P$. aeruginosa in surface water and to screen its antibiotic resistance pattern. Finally, the resistant isolates are classified according to extended spectrum beta-lactamase production.

\section{Methods and Materials}

\subsection{Sample Collection (Time and Zone)}

The overall study was carried out over a period of eight months. All of the samples were collected in two phases: firstly, at the initial period on summer season and secondly, at the late period on winter season. Overall Dhaka city was sub-divided into north and south zone to facilitate the collection of sample. Water samples were collected from various ponds, lakes and rivers in Dhaka city. And all the samples were stored in sterile bottle at 40 $\mathrm{C}$ until processing. Within 4 hour all of the samples were processed accordingly.

\subsection{Isolation of Bacteria}

$5 \mathrm{ml}$ of water sample was added to $20 \mathrm{ml}$ Baumann's enrichment medium and incubated overnight without 
shaking at $300^{\circ} \mathrm{C}$. Then the sample was streaked for isolation on Mac Conkey agar and incubated at $300^{\circ} \mathrm{C}$ for 18 - 22 hrs. Predominant colorless colony for Pseudomonas aeruginosa on MacConkey agar was selected for characterization.

\subsection{Characterization of Gram Negative Bacteria}

According to the society of American Bacteriologists [17], colorless colony on Mac Conkey agar was observed for morphological characteristics and considered for identification. Several Physiological (Oxidase, catalase tests) and biochemical tests [Citrate Utilization, Kligler's iron agar (KIA), Motility Indole Urea (MIU), Methyl Red (MR), Voges-Proskauer (VP) Test, Gram staining and Microscopic examination, were carried out for characterization of Pseudomonas [18]. For indentification of Pseudomonas aeruginosa, suspicious isolates were grown in nutrient broth at $420^{\circ} \mathrm{C}$ in $4 \mathrm{hrs}$ [18]. Positive growth was identified as Pseudomonas aeruginosa.

\subsection{Determination of Antimicrobial Susceptibility Test by Disk Diffusion Method}

Antimicrobial susceptibility tests were performed to measure the ability of an antibiotic to inhibit bacterial growth in vitro by disc diffusion. This test was performed by modified Kirby-Bauer method [19]. The result of the test was interpreted according to the Clinical Laboratory Standard Institute guidelines (CLSI) in 2008 [20]. Bacterial culture from nutrient agar was inoculated into $3 \mathrm{ml}$ Mueller-Hinton broth with a sterile loop. The broth was incubated at $370^{\circ} \mathrm{C}$ for 3 to 4 hours. Turbidity of the broth culture was adjusted to a MacFarland 0.5 standard $(1.5 \times 108 \mathrm{CFU} / \mathrm{ml})$. MacFarland 0.5 was prepared by adding $0.5 \mathrm{ml}$ of $0.048 \mathrm{M} \mathrm{BaCl} 2\left(1.75 \% \mathrm{BaCl}_{2}\right.$, $\left.2 \mathrm{H}_{2} \mathrm{O}\right)$ to $99.5 \mathrm{ml}$ of $0.36 \mathrm{NH}_{2} \mathrm{SO}_{4}(1 \%)$ with Mueller Hinton broth. When the proper density was achieved, a sterile cotton swab was submerged in the suspension, lift out of the broth, and excess fluid was removed by pressing and rotating the swab against the wall of the tube. The swab was then used to inoculate the entire surface of the supplemented Mueller-Hinton agar plate three times, rotating the plate 900 between each inoculation. The inoculated plate was allowed to dry (usually taking only a few minutes) before the discs was placed on the plate. Commercial antibiotic discs (supplied by Becton-Dickinson) were placed on the agar plates. The plates containing the discs were incubated at $370^{\circ} \mathrm{C}$ for 16 to 18 hours. Only five distinct antibiotics, Gentamicin $(10$ $\mu \mathrm{g})$, Ciprofloxacin $(5 \mu \mathrm{g})$, Ampicillin $(10 \mu \mathrm{g})$, Tetracycline $(30 \mu \mathrm{g})$, Colistin $(10 \mu \mathrm{g})$, Polymixin-B $(300 \mu \mathrm{g})$ and Co-trimoxazole $(25 \mu \mathrm{g})$ were used for testing the resistant pattern of $\mathrm{P}$. aeruginosa. Estimated the susceptibility of the strain after overnight incubation, the diameter of each zone of inhibition was measured with a scale (in $\mathrm{mm})$.

\subsection{Detection of ESBLs Producing Isolates}

The isolates those are resistant to Ampicillin were screened for ESBL production by the double disc synergy test [21]. An agar plate was inoculated as described for the standard disc diffusion method. Antibiotic disc of four different Cephalosporine (Cefotaxime (30 $\mu \mathrm{g})$, Ceftadizime (30 $\mu \mathrm{g})$, Imepenem $(10 \mu \mathrm{g})$ and Aztreanam (30 $\mu \mathrm{g}))$ were placed at center to center distances of $30 \mathrm{~mm}$ apart from an Ampicillin clavunic acid (20/10 $\mu \mathrm{g})$. A clear extension of greater than $5 \mathrm{~mm}$ inhibition zone between any of the four Cephalosporins towards the disc containing Clavulanic acid was interpreted as positive for ESBL production.

\section{Results}

\subsection{Prevalence Rate}

52 water samples were collected from different water sources like ponds, lakes and river of Dhaka city. Around them thirty two samples were found positive for P. aeruginosa. Out of the 32 positive isolates, 13 were from ponds, 16 from lakes and 3 from river. The highest isolation rates of $P$. aeruginosa was found in lake (69.5\%) followed by river $(60 \%)$ and ponds $(54.1 \%)$. The prevalence rates of $P$. aeruginosa recovered from different surface water is given in Table 1 .

\subsection{Antibiotic Resistance Pattern}

A number of clinically important antibiotics such as Gentamycin, Ciprofloxacin, Ampicillin, Tetracycline, Polymixin-B, Colistin and Co-trimoxazole were used to test the resistant pattern of Pseudomonas aeruginosa. It 
Table 1. Isolation rates of $P$. aeruginosa recovered from different surface water.

\begin{tabular}{ccc}
\hline Site of collection & No. of sample examined & No. of positive isolates (\%) \\
\hline Ponds & 24 & $13(54.1 \%)$ \\
Lake & 23 & $16(69.5 \%)$ \\
River & 5 & $3(60 \%)$ \\
Total & 52 & $32(61.5 \%)$ \\
\hline
\end{tabular}

was found that the isolates were mostly resistant to Ampicillin (100\%), Tetracycline (93.7\%) and Gentamycin $(93.7 \%)$. On the other hand, they were moderately resistant to co-trimoxazole $(71.8 \%)$ but marginally resistant to polymixin-B (21.8\%) and Colistin (12.5\%). Paradoxically, the isolates were completely sensitive to third generation antibiotic Ciprofloxacin (100\%) (Figure 1 and Figure 2).

\subsection{Evaluation of Resistance Pattern}

In the extension of present study, all of the isolates were found completely resistant to the third generation antibiotics Cephalosporins (Cefotaxime and Ceftazidime). Ironically, they were found susceptible to ciprofloxacin, Imipenem and Aztreonam. Ampicillin clavunice acid was also tested to determine ESBL production by the isolates. Surprisingly, the resistant isolates were also found resistant to Ampicillin clavunic acid. All of these test result suggest that $100 \%$ isolates were positive for ESBL production and according to the Ambler and Bonnet classification [22] [23] isolated Pseudomonas aeruginosa is under the group of AmpC type (Table 2).

\section{Discussions}

P. aeruginosa is one of the important bacterial pathogens ubiquitous in different environmental sample. Despite advances in medical and surgical care and introduction of wide variety of antimicrobial agents in animal and agriculture, resistant isolates increases dramatically in environmental surface water. Therefore, different water sources could create a widespread pool of resistant microbes from which resistance could be transferred back into human and animal disease organisms. So the present study was carried out to find out the proportion of the resistance isolates in surface water and to screen their antibiotic resistance pattern.

In the present study, 52 water samples were collected from three different water sources in Dhaka city like ponds, rivers and lakes. Around them 32 samples were found to be positive for P. aeruginosa isolates. The highest isolation rates of $P$. aeruginosa was found in lake $(69.5 \%)$ followed by $60 \%$ in river and ponds were for $54.1 \%$. The isolation rate of different water samples could vary due to source and size of sample as well as time and duration of sample collection. However, in Bangladesh the average isolation rate from different surface water for $P$. aeruginosa is $61.5 \%$ which is relatively higher than its neighboring country India that was found $45.45 \%$ by Sivaraj et al. in 2011 [24].

This current study mainly focused to the characterization of antibiotic resistant pattern of $P$. aeruginosa isolated from different sources of surface water. Here, mostly used anti-pseududomonal agents in Bangladesh were tested against Pseudomonas aeruginosa isolates to screen their resistant pattern. Those antibiotics were Penicillin (Ampicillin), Carbapenem (Imipenem), third generation cephalosporins (Cefotaxime, Ceftadizime and Aztreonam), Polymixin (Polymixin-B and Colistin), Sulphonamide (Co-trimoxazole), Tetracycline, aminoglycosides (gentamycin), Quinolones (Ciprofloxacin), and Ampicillin combination (Ampicillin clavunic acid).

According to the Disk Diffusion Method, isolates were found 100\% resistant to Ampicillin and similar result was found also in Pakisan in 2010 for clinical samples [25]. But an Indian report conducted by Krishnakumar et al. (2012) [26] found 50\% Ampicillin resistant isolates from clinical sample.

The present study detected $93.7 \%$ isolates were resistant to both Tetracycline and Gentamycin antibiotic. An elevated rate of resistant was also found in 100\% in Egypt [27], 99\% in Pakistan [25] for Tetracyline.

Two Indian study in 2012 [28] [29] showed 63\% isolated from clinical samples were resistant to Gentamycin. Even high resistance to Gentamycin was also found in India, Iran, and Pakistan [30]-[32]. A study performed by Rashid et al. showed $77.3 \%$ in resistance to same antibiotic in clinical sample in 2012 [33]. A paper accomplished by Ahmed et al. in 2013 found 43.9\% Gentamycin resistant isolates in clinical sample in Egypt [27]. According to Indian, Pakistan, the proportion of isolates in this study was more resistant to Gentamycin. 


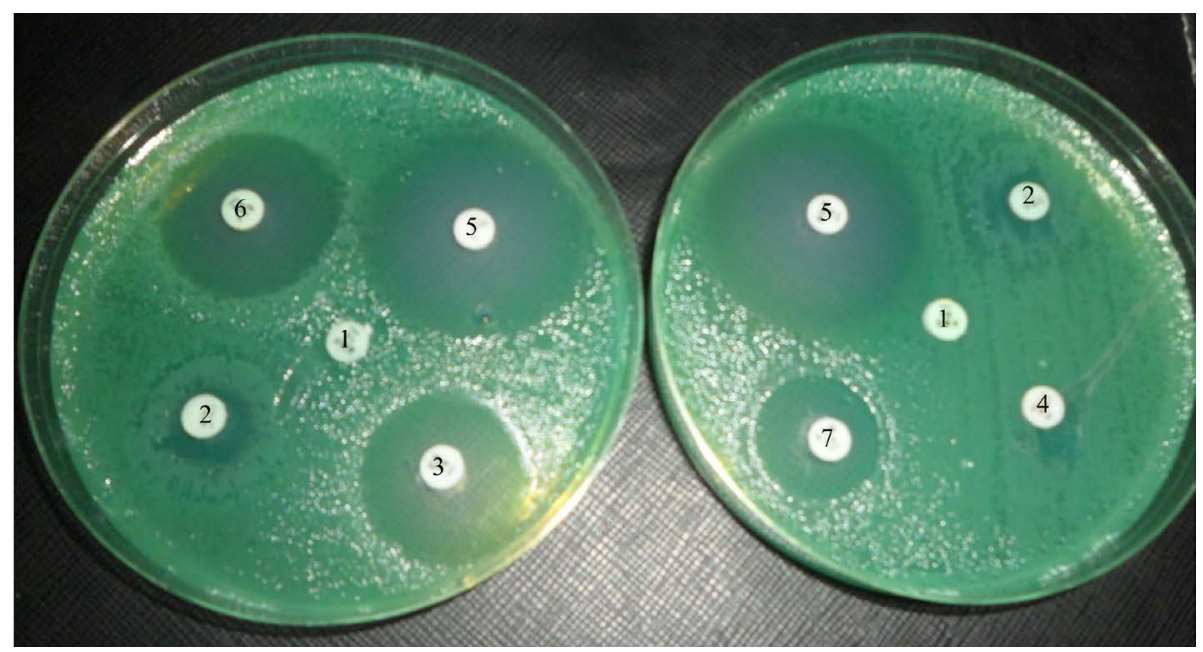

Figure 1. Antibiotic resistance pattern in $P$. aeruginosa disc 1 of both plates represents for Amplicillin that showed maximum resistance (100\%) followed by discs 2 and 4 of second plate represent for Tetracycline and Gentamycin showed resistance almost similar to Ampicillin. Otherwise, discs 3 and 6 of the first plate represent for Colistin and Polymixin-B show immense sensitivity to $P$. aeruginosa. And disc 5 of both plate for Ciprofloxacin showed the highest sensitivity.

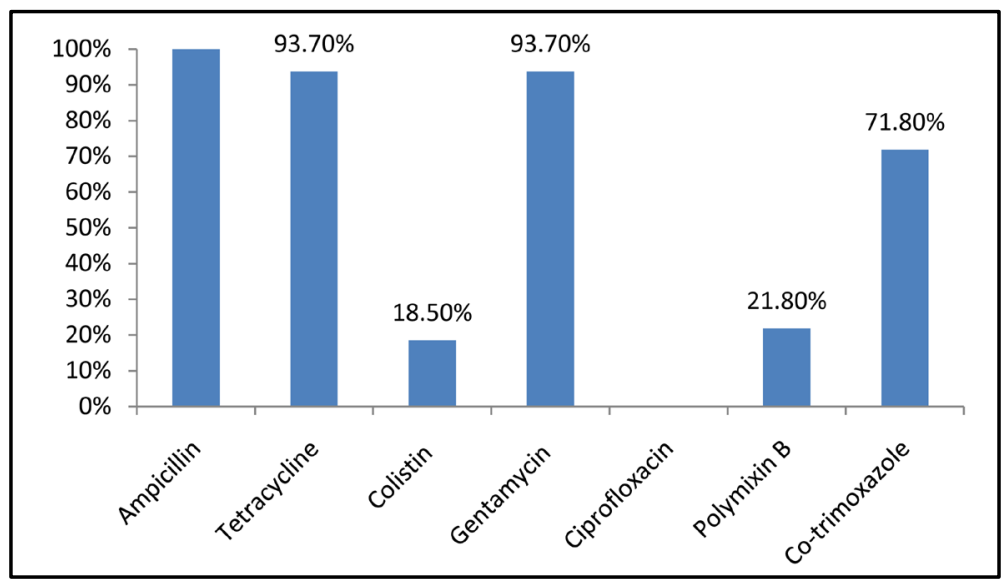

Figure 2. Antimicrobial resistance pattern of $P$. aeruginosa isolated from surface water in Dhaka City.

Table 2. Characterization of ampicillin resistant isolates by Double Disk Synergy Test (DDST).

\begin{tabular}{lllllllll}
\hline & \multicolumn{4}{c}{ Susceptibility to } & & Types of Beta- \\
Isolates $(\mathrm{N}=32)$ & Ampicillin & Ampicillin clavunic acid & Cefotaxime & Ceftadizime & Aztreonam & Imipenem & lactamases production \\
\hline P. aeruginosa $(100 \%)$ & $\mathrm{R}$ & $\mathrm{R}$ & $\mathrm{R}$ & $\mathrm{R}$ & $\mathrm{S}$ & $\mathrm{S}$ & AmpC (class C/group 1) \\
\hline
\end{tabular}

$71.8 \%$ resistant to Co-trimoxazole was found in this study which was slightly lower than $93.5 \%$ resistant isolates shown in study of Rashid et al. [33]. Clinical isolates (95.4\%) were resistant to to co-trimoxazole in Iran shown in 2012 [31]. The present study found isolates $(21.8 \%)$ resistant to Polymixin-B and approximately same percentage $(20 \%)$ of isolates were also found to be resistant in the study of Angadi [29].

$12.5 \%$ resistant to Colistin was found in this work where an Indian study of Angadi et al., found $15.2 \%$ resistance [29].

The isolates of this present study were found to be completely sensitive to third generation antibiotic Ciprofloxacin (100\%). But a completely different situation was found by Rashid et al. (2012) in also in Bangladesh. 
They isolated Pseudomonas aeruginosa from clinical sample where they saw, $75.5 \%$ isolates were resistant to ciprofloxacin [33]. Rajat and his co-worker found that isolates from clinical sample were resistant to Cipropfloxacin (49\%) in India in 2012 [28].

The present study was also extended to classify the isolated $P$. aeruginosa according to beta lactamases production. Double disc synergy test suggests that all of the isolates were susceptible to Imipenem and Aztreonam and were completely resistant to third generation antibiotic Cephalosporins (Cefotaxime and Ceftazidime). An Indian study in 2012 showed that $86 \%$ and $61 \%$ isolates susceptible to Imepenem and Aztrenam respectively [28]. A study in 2007 in Bangladesh, found 86\% resistant isolates to cefrtiaxone and ceftazidime [33]. Besides, the resistant isolates were also found to double resistant against Ampicillin clavunic acid. The same result was observed in clinical samples in India [26].

These DDST suggested that the isolated $P$. aeruginosa were competent for ESBL production. And according to the Ambler and Bonnet classification [22] [23], the isolated Pseudomonas aeruginosa is confirmed as the member of AmpC type (class C/ group 1). In 2010, 59.4\% clinical isolates were found to be positive for AmpC beta-lactamase production in India [34]. All the isolates were Multidrug Resistant (MDR) ( $>3$ antibiotic). 8\% MDR isolates from water sample was recorded in India [24]. It was reported isolates from clinical samples that resistance to multi drug was $100 \%$ in Iran [35], 20.69\% in Nepal [36], 19.6\% in Malaysia [37].

It is evident from the study that now a day's $P$. aeruginosa is becoming less sensitive to cephalosporins, aminoglycosides and other B-lactamase inhibitors. And the surface water is somehow contaminated with such antibiotic resistant $P$. aeruginosa and through the water systems antibiotic resistance transferred to human and animal. Thereby, the living organism gradually becomes multidrug resistant. To prevent the spread of the resistant bacteria, it is critically important to have strict antibiotic policies while surveillance program for multidrug resistant organisms and infection control procedures need to be implemented.

\section{Conclusion}

Different sources of environment can play an important role in the spreading of P. aeruginosa [21]. Surface water is one of them, which is currently found to be affluent with multi-drug resistance $P$. aeruginosa. The present study suggests that the resistance of $P$. aeruginosa increases due to the uncontrolled usage and disposing of antibiotics in the environment. Treatment may fail to recover by constant contact of resistance isolates. So, we should be aware of the emergence and spread of multi-resistant isolates of clinically important bacteria.

\section{References}

[1] Favero, M.S., Carson, L.A., Bond, W.W. and Petersen, N.J. (1971) Pseudomonas aeruginosa: Growth in Distilled Water. Science, 173, 836-838. http://dx.doi.org/10.1126/science.173.3999.836

[2] Remington, J.S and Schimpff, S.C. (1981) Please Don't Eat Salads. The New England Journal of Medicine, 304, 433435. http://dx.doi.org/10.1056/NEJM198102123040730

[3] Joklik, K.W., Willet, P.H., Amos, B.D. and Catherine, M.W. (1992) Pseudomonas. Zinser Microbiology, 20, 576-583.

[4] Berrouane, F.Y., McNutt, L., Buschellman, B.J., Rhomberg, P.R., Sanford, M.D., Hollis, R.J., Pfaller, M.A. and Herwaldt, L.A. (2000) Outbreak of Severe Pseudomonas aeruginosa Infection Caused by Contaminated Drains in a Whirlpool Bathtubs. Clinical Infectious Diseases, 31, 1331-1337. http://dx.doi.org/10.1086/317501

[5] Anthony, M., Rose, B., Pegler, M.B., Elkins, M. Service, H., Thamotharampillai, K., Watson, J., Robinson, M., Bye, P., Merlino, J. and Harbour, C. (2002) Genetic Analysis of Pseudomonas aeruginosa Isolates from the Sputa of Australian Adult Cystic Fibrosis Patients. Journal of Clinical Microbiology, 40, 2772-2778. http://dx.doi.org/10.1128/JCM.40.8.2772-2778.2002

[6] Kato, K., Iwai, S., Kumasaka, K., Horikoshi, A.,Inada, S., Inamatsu, T., Ono, Y., Nishiya, H., Hanatani, Y., Na rita, T., Sekino, H., and Hayashi, I. (2001) Survey of Antibiotic Resistance in Pseudomonas aeruginosa by the Tokyo Johoku Association of Pseudomonas Studies. Journal of Infection and Chemotherapy, 7, 258-262. http://dx.doi.org/10.1007/s101560170024

[7] Aeschlimann, J. (2003) The Role of Multidrug Efflux Pumps in the Antibiotic Resistance of Pseudomonas aeruginosa and Other Gram-Negative Bacteria. Pharmacother, 23, 916-924. http://dx.doi.org/10.1592/phco.23.7.916.32722

[8] Lister, P.D., Wolter, D.J. and Hanson, N.D. (2009) Antibacterial-Resistant Pseudomonas aeruginosa: Clinical Impact and Complex Regulation of Chromosomally Encoded Resistance Mechanisms. Clinical Microbiology Reviews, 22, 582-610. http://dx.doi.org/10.1128/CMR.00040-09

[9] Gales, A.C., Jones, R.N., Turnidge, J., Rennie, R. and Ramphal, R. (2001) Characterization of Pseudomonas aerugi- 
nosa Isolates: Occurrence Rates, Antimicrobial Susceptibility Patterns, and Molecular Typing in the Global SENTRY Antimicrobial Surveillance Program, 1997-1999. Clinical Infectious Diseases, 32, S146-S155. http://dx.doi.org/10.1086/320186

[10] Carmeli, Y., Troillet, N., Karchmer, A.W. and Samore, M.H. (1999) Health and Economic Outcomes of Antibiotic Resistance in Pseudomonas aeruginosa. JAMA Internal Medicine 159, 1127-1132. http://dx.doi.org/10.1001/archinte.159.10.1127

[11] Goossens, H., Ferech, M., Vander Stichele, R. and Elseviers, M. (2005) Outpatient Antibiotic Use in Europe and Association with Resistance: A Cross-National Database Study. Lancet, 365, 579-587. http://dx.doi.org/10.1016/S0140-6736(05)17907-0

[12] D’Agata, FEMC (2000) Antibiotic Resistance and Exposure to Different Generation Cephalosporins. The New England Journal of Medicine, 28, 2678-2681.

[13] Fridkin, S., Steward, C., Edwards, J., Pryor, E., McGowan, J., Archibald, L., et al. (1999) Surveillance of Antimicrobial Use and Antimicrobial Resistance in United States Hospitals: Project ICARE Phase 2. Clinical Infectious Diseases, 29, 245-252. http://dx.doi.org/10.1086/520193

[14] Paterson, D., Mulazimoglu, L., Casellas, J., Ko, W., Goossens, H., Von Gottberg, A., et al. (2005) Epidemiology of Ciprofloxacin Resistance and Its Relationship to Extended-Spectrum Beta-Lactamase Production in Klebsiella pneumoniae Isolates Causing Bacteremia. Clinical Infectious Diseases, 30, 473-478. http://dx.doi.org/10.1086/313719

[15] Bradford, P. (2001) Extended-Spectrum Beta-Lactamases in the 21st Century: Characterization, Epidemiology, and Detection of This Important Resistance Threat. Clinical Microbiology Reviews, 14, 933-951. http://dx.doi.org/10.1128/CMR.14.4.933-951.2001

[16] Giraud-Morin, C. and Fosse, T. (2003) A Seven-Year Survey of Klebsiella pneumoniae Producing TEM-24 ExtendedSpectrum Beta-Lactamase in Nice University Hospital (1994-2000). Journal of Hospital Infection, 54, 25-31. http://dx.doi.org/10.1016/S0195-6701(03)00038-0

[17] Pelczar, M., Reid, R. and Chan, E. (1957) Microbiology. T.M.H. Edition, Tata McGraw-Hill Publishing Company Ltd., Noida.

[18] Cheesbrough, M. (2006) District Laboratory Practice in Tropical Countries. Part 2. Cambridge University Press, Cambridge, 434.

[19] Bauer, A., Kirby, W., Sherris, J. and Turck, M. (1966) Antibiotic Susceptibility Testing by a Standardized Single Disk Method. American Journal of Clinical Pathology, 45, 493-496.

[20] Clinical and Laboratory Standards Institute (2008) Performance Standards for Antimicrobial Susceptibility Testing; Eighteenth Informational Supplement (Document M100-S18). Vol. 4, The Clinical and Laboratory Standards Institute, Wayne, 354.

[21] Ho, P., Chow, K., Yuen, K., Ng, W. and Chau, P. (1998) Comparison of a Novel, Inhibitor-Potentiated Disc-Diffusion Test with Other Methods for the Detection of Extended-Spectrum Beta-Lactamases in Escherichia coli and Klebsiella pneumoniae. Journal of Antimicrobial Chemotherapy, 42, 49-54. http://dx.doi.org/10.1093/jac/42.1.49

[22] Ambler, R., Coulson, A., Frere, J., Ghuysen, J., Oris, J., Forsman, R., Levesques, R., Tirabi, G. and Waley, S. (1991) A Standard Numbering Scheme for the Class A Beta Lactamase. Biochemical Journal, 276, 269-272.

[23] Bonnet, R. (2004) Growing Group of Extended Spectrum $\beta$-Lactamase: The CTX-M Enzymes. Antimicrobial Agents and Chemotherapy, 48, 1-14. http://dx.doi.org/10.1128/AAC.48.1.1-14.2004

[24] Sivaraj, S., Murugesan, P., Muthuvelu, S., Purusothaman, S. and Silambarasan, A. (2011) Comparative Study of Pseudomonas aeruginosa Isolate Recovered from Clinical and Environmental Samples against Antibiotics. International Journal of Pharmacy \& Pharmaceutical Science, 4, 103-110.

[25] Anjum, F. and Mir, A. (2010) Susceptibility Pattern of Pseudomonas aeruginosa against Various Antibiotics. African Journal of Microbiology Research, 4, 1005-1012.

[26] Krishnakumar, S., Rajan, R.A., Babu, M.M. and Bai, V.D.M. (2012) Antimicrobial Susceptibility Pattern of Extended Spectrum of Beta Lactamase (ESBL) Producing Uropathogens from Pregnant Women. Indian Journal of Medicine and Healthcare, 1, 188-192.

[27] Mahmoud, A.B., Zahran, W.A., Hindawi, G.R., Labib, A. and Galal, R. (2013) Prevalence of Multidrug-Resistant Pseudomonas aeruginosa in Patients with Nosocomial Infections at a University Hospital in Egypt, with Special Reference to Typing Methods. Journal of Virology \& Microbiology, 2013, 10-13.

[28] Rakesh, M.R., Govind, N.L., Kalpesh, M., Rosy, P., Kanu, P. and Vegad, M.M. (2012) Antibioitc Resistance Pattern in Pseudomonas aeruginosa Species Isolated at a Tertiary Care Hospital, Ahmadabad. National Journal of Medical Research, 2, 156-159.

[29] Angadi, K.M., Kadam, M., Modak, M.S., Bhatavdekar, S.M., Dalal, B.A., Jadhavvar, S.R., Tolpadi, A.G., Thakkar, V. 
and Shah, S.R. (2012) Detection of Antibiotic Resistance in Pseudomonas aeruginosa Isolates with Special Reference to Metallo $\beta$-Lactamases from A Tertiary Care Hospital in Western India. International Journal of Microbiology Research, 4, 295-298.

[30] Raja, N.S. and Singh, N.N. (2007) Antimicrobial Susceptibility Pattern of Clinical Isolates of Pseudomonas aeruginosa in a Tertiary Care Hospital. Journal of Microbiology, Immunology and Infection, 40, 45-49.

[31] Saderi, H., Lotfalipour, H., Owlia, P. and Salimi, H. (2010) Detection of Metallo- $\beta$-Lactamase Producing Pseudo monas aeruginosa Isolated from Burn Patients in Tehran, Iran. Laboratory Medicine, 41, 609-612. http://dx.doi.org/10.1309/LMQJF9J3T2OAACDJ

[32] Naqvi, Z.A., Hashmi, K., Rizwan, Q. and Kharal, S.A. (2005) Multi Drug Resistant Pseudomonas aeruginosa: A Nosocomial Infection Threat in Burn Patients. Pakistan Journal of Pharmacology, 22, 9-15.

[33] Rashid, A., Chowdhury, A., Rahman, S.H.Z., Begum, S.A. and Muazzam, N. (2007) Infections by Pseudomonas aeruginosa and Antibiotic Resistance Pattern of the Isolates from Dhaka Medical College Hospital. Bangladesh Journal of Medical Microbiology, 1, 48-51.

[34] Upadhyay, S., Sen, M.R. and Bhattacharjee, A. (2010) Presence of Different Beta-Lactamase Classes among Clinical Isolates of Pseudomonas aeruginosa Expressing AmpC Beta-Lactamase Enzyme. Journal of Infection in Developing Countries, 4, 239-242.

[35] Moazami-Goudarzi, S. and Eftekhar, F. (2013) Assessment of Carbapenem Susceptibility and Multidrug-Resistance in Pseudomonas aeruginosa Burn Isolates in Tehran. Jundishapur Journal of Microbiology, 6, 162-165.

[36] Anil, C. and Shahid, R.M. (2013) Antimicrobial Susceptibility Patterns of Pseudomonas aeruginona Clinical Isolates at a Tertiary Care Hospital in Kathmandu, Nepal. Asian Journal of Pharmaceutical and Clinical Research, 6, 235-238.

[37] Pathmanathan, S.G., Samat, N.A. and Mohamed, R. (2009) Antimicrobial Susceptibility of Clinical Isolates of Pseudomonas aeruginosa from a Malaysian Hospital. Malaysian Journal of Medical Sciences, 16, 27-32. 
Scientific Research Publishing (SCIRP) is one of the largest Open Access journal publishers. It is currently publishing more than 200 open access, online, peer-reviewed journals covering a wide range of academic disciplines. SCIRP serves the worldwide academic communities and contributes to the progress and application of science with its publication.

Other selected journals from SCIRP are listed as below. Submit your manuscript to us via either submit@scirp.org or Online Submission Portal.
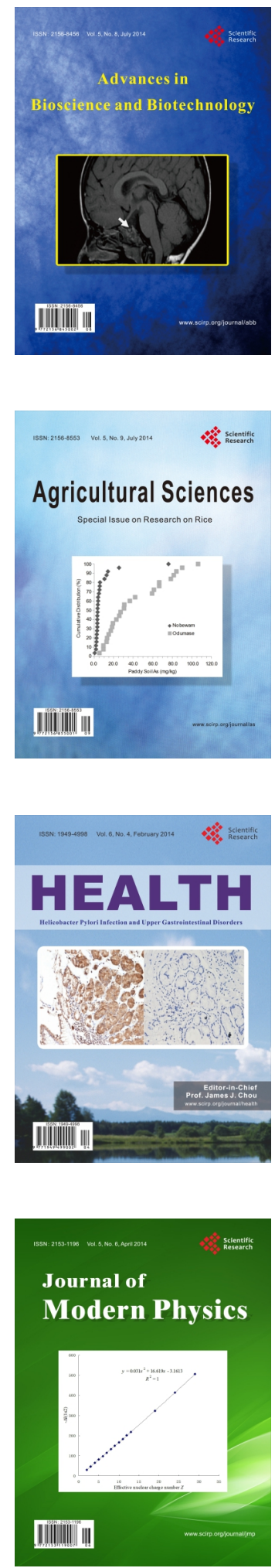
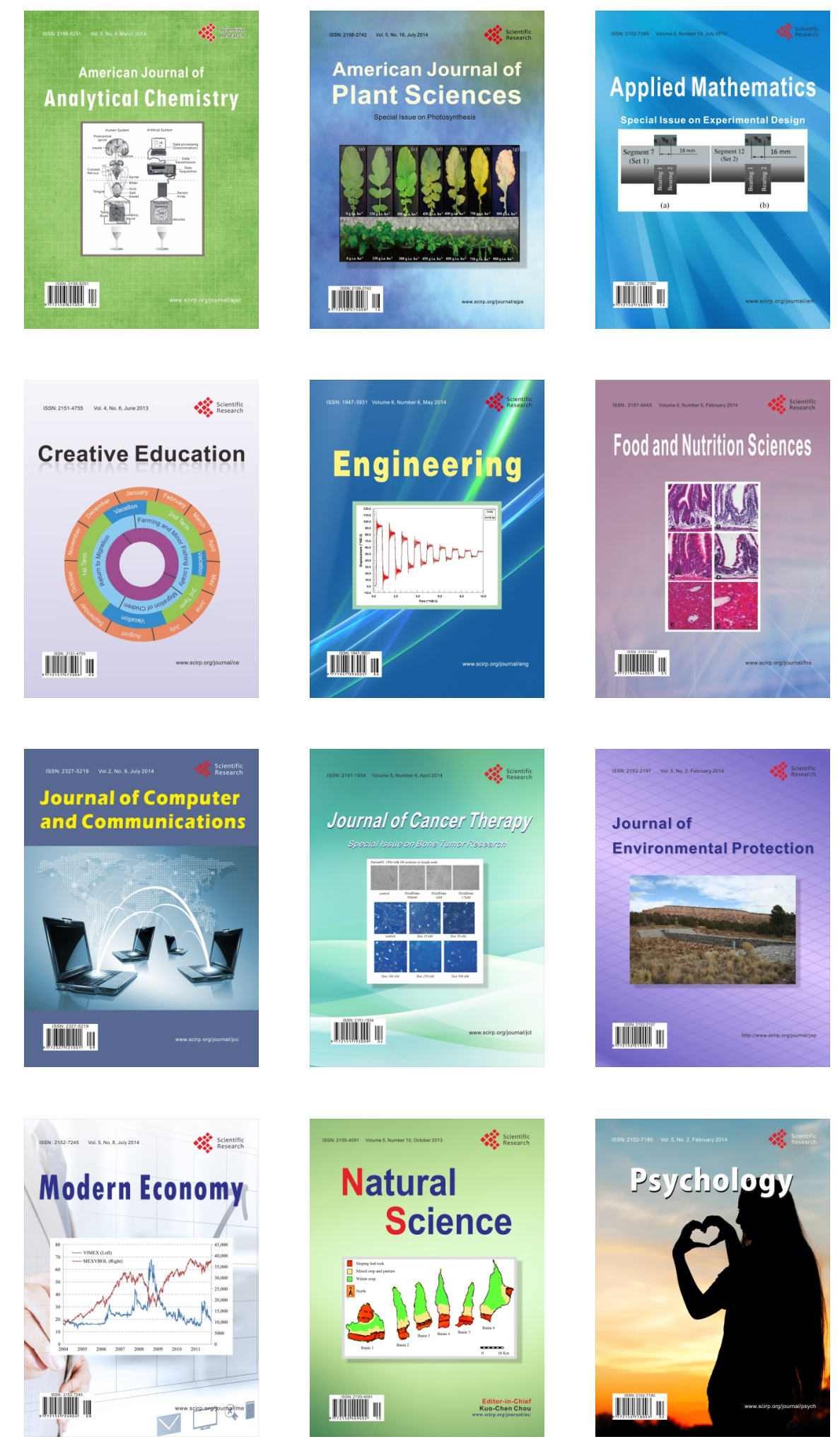